\title{
Prevalence of Clostridium difficile infection and colonization in a tertiary hospital and elderly community of North-Eastern Peninsular Malaysia
}

\author{
N. H. ZAINUL ${ }^{1} \uparrow$, Z. F. MA ${ }^{1,2} \dagger, A$ A. BESARI ${ }^{1}$, H. SITI ASMA ${ }^{3}$, R. A. RAHMAN ${ }^{3}$, \\ D. A. COLLINS ${ }^{4}$, N. HAMID ${ }^{1}$, T. V. RILEY ${ }^{4,5,6}$ AND Y. Y. LEE ${ }^{1 *}$ \\ ${ }^{1}$ Department of Medicine, School of Medical Sciences, Universiti Sains Malaysia, Kubang Kerian, Kelantan, Malaysia \\ ${ }^{2}$ Department of Public Health, Xi'an Jiaotong-Liverpool University, Suzhou, China \\ ${ }^{3}$ Department of Medical Microbiology and Parasitology, School of Medical Sciences, Universiti Sains Malaysia, \\ Kubang Kerian, Kelantan, Malaysia \\ ${ }^{4}$ School of Medical and Health Sciences, Edith Cowan University, Perth, Australia \\ ${ }^{5}$ Veterinary and Life Sciences, Murdoch University, Perth, Australia \\ ${ }^{6}$ Department of Microbiology, PathWest Laboratory Medicine (WA), Perth, Australia
}

Received 27 March 2017; Final revision 1 July 2017; Accepted 14 August 2017; first published online 11 September 2017

\section{SUMMARY}

Little is known about Clostridium difficile infection (CDI) in Asia. The aims of our study were to explore (i) the prevalence, risk factors and molecular epidemiology of CDI and colonization in a tertiary academic hospital in North-Eastern Peninsular Malaysia; (ii) the rate of carriage of C. difficile among the elderly in the region; (iii) the awareness level of this infection among the hospital staffs and students. For stool samples collected from hospital inpatients with diarrhea $(n=76)$ and healthy community members $(n=138), C$. difficile antigen and toxins were tested by enzyme immunoassay. Stool samples were subsequently analyzed by culture and molecular detection of toxin genes, and PCR ribotyping of isolates. To examine awareness among hospital staff and students, participants were asked to complete a self-administered questionnaire. For the hospital and community studies, the prevalence of non-toxigenic $C$. difficile colonization was $16 \%$ and $2 \%$, respectively. The prevalence of CDI among hospital inpatients with diarrhea was $13 \%$. Out of 22 C. difficile strains from hospital inpatients, the toxigenic ribotypes 043 and 017 were most common (both 14\%). In univariate analysis, $C$. difficile colonization in hospital inpatients was significantly associated with greater duration of hospitalization and use of penicillin (both $P<0.05)$. Absence of these factors was a possible reason for low colonization in the community. Only $3 \%$ of 154 respondents answered all questions correctly in the awareness survey. C. difficile colonization is prevalent in a Malaysian hospital setting but not in the elderly community with little or no contact with hospitals. Awareness of CDI is alarmingly poor.

Key words: Awareness, Clostridium difficile, elderly, epidemiology, patients, prevalence, risk factors.

\section{INTRODUCTION}

Clostridium difficile, a spore-forming and Grampositive bacterium produces two major diarrhea- producing toxins, which are toxin $\mathrm{A}(\mathrm{TcdA})$ and toxin $\mathrm{B}(\mathrm{TcdB})[1]$. The genes for these toxins, $t c d A$ and $t c d B$, are not carried by non-toxigenic $C$. difficile

\footnotetext{
* Author for correspondence: Y. Y. Lee, School of Medical Sciences, Universiti Sains Malaysia, Kubang Kerian, Kelantan, Malaysia. (Email: yylee@usm.my)

$\dagger$ Joint first author.
} 
strains. Completely non-toxigenic strains of $C$. difficile do not cause disease. Some strains of $C$. difficile produce a third toxin, a binary toxin (CDT), the significance of which remains controversial [2]. Since $C$. difficile is one of the most common nosocomial infections worldwide [3-5], colonization with toxigenic or non-toxigenic $C$. difficile is higher in hospitalized patients than in healthy adults (i.e. $20-40 \%$ vs. $2-$ $3 \%$ [6]. More than $90 \%$ of $C$. difficile infection (CDI) occurs after or while patients on antimicrobial therapy [7]. On the other hand, asymptomatic colonization in the adult and elderly communities has been more variable with prevalence ranging from $0 \%$ to $51 \%[8]$.

The prevalence of CDI has increased significantly over the past two decades [9]. A Canadian nosocomial surveillance project reported a fourfold increase in the incidence of CDI from $22 \cdot 2$ cases per 100000 population to 92.2 cases per 100000 population from 1998 until 2006 [10]. Moreover, certain strains of $C$. difficile cause more severe infection, especially ribotypes (RTs) 027 and 078 [11]. RTs 027 and 078 produce TcdA and TcdB in higher quantities than other strains, and a third binary toxin (CDT) probably due to the mutated TcdC protein [12], although this may be irrelevant in terms of disease pathogenesis. Diagnosis of CDI requires detection of toxin in diarrheal stool [13]. The quickest and cheapest method of toxin detection is by enzyme immunoassay (EIA). Otherwise, culture and/or PCR detection of $t c d B$ are effective ways to detect toxigenic $C$. difficile, but may not differentiate colonized patients from infected patients.

Although data on the epidemiology of CDI in North America and Europe are plentiful, limited data are available for the rest of the world, particularly Asia [14]. This is because comprehensive diagnostic testing for $C$. difficile is lacking in many Asian hospitals. In addition, it is perceived that awareness of CDI among physicians especially outside the Western hemisphere is low [15]. There have been only five papers published on $C$. difficile in Malaysia and, of these, three were published prior to 1997 [16-18]. Although Hassan et al. reported a hospital prevalence of $14 \%$ by toxin EIA [19], Syuhada et al. reported a lower prevalence of $6 \%$ [20], and the differences might be because of low awareness and testing of CDI, or differences in patient populations. No data on carriage of $C$. difficile by the elderly in the community are available in Malaysia but these data may be important to help explain the high prevalence of CDI reported in hospitals.
Therefore, the aims of our study were: (1) to investigate the prevalence, associated risk factors and ribotyping of $C$. difficile in hospitalized patients; (2) to determine the carriage rate of $C$. difficile and associated risk factors in an elderly community and (3) to explore awareness of CDI among staff and students in a tertiary academic hospital.

\section{MATERIALS AND METHODS}

\section{Hospital study population}

Inpatients from the only tertiary academic hospital (Hospital Universiti Sains Malaysia, Kota Bharu, Kelantan) in the north-eastern region of Peninsular Malaysia were recruited from April 2015 to September 2015. They were aged between 18 and 80 years, with or without antibiotic-associated diarrhea within $48 \mathrm{~h}$ of hospital admission (either the acute-care hospital or long-term care facility that provided skilled nursing care for $\geqslant 1$ overnight stay) and those suspected to have CDI. The duration of hospitalization was defined as time from admission until discharge. The $48 \mathrm{~h}$ was selected according to a surveillance definition paper by McDonald et al. [21]. Those who were unable to provide stool samples due to medical or psychiatric reasons were excluded from study. Data entry collection forms for eligible participants were completed by interviewing or reviewing their case notes, and radiological and microbiological data.

\section{Community study population}

Elderly people aged between 60 and 90 years were recruited between July 2015 and September 2015 from two randomly selected communities of Tumpat and Kota Bharu districts and from an aged residential care facility in Kota Bharu. Exclusion factors were a history of healthcare contact within the 12 weeks prior to enrolment, or previous hospitalization due to $C$. difficile in the last 28 days, or current suspicion of CDI and inability to provide stool sample. Questionnaires were completed through interviews of eligible elderly participants by a trained research assistant.

\section{Awareness survey population}

University hospital staff (i.e. doctors, non-clinical medical professionals and paramedics) and students (i.e. undergraduates and postgraduates) who were involved with in-hospital patient care were surveyed 
during a workshop. They were asked to answer a Malay language structured questionnaire comprising of five multiple-choice questions with a single answer, as reported by Mavros et al. [15]. Briefly, the first four questions were related to CDI awareness which included knowledge of likelihood of treatment failure or recurrence after first episode of treated $C$. difficileassociated diarrhea (CDAD), knowledge of approximate percentage of antibiotic-associated colitis due to $C$. difficile, knowledge of antibiotic(s) that put(s) patient at risk for CDAD and knowledge of the type of patients with diarrhea that may have CDAD. The last question asked about the respondent estimation of CDAD frequency and morbidity in his or her current clinical practice.

\section{Laboratory analysis}

Once stool samples were collected from hospital inpatients and community participants, they were stored at $2-8{ }^{\circ} \mathrm{C}$ in the microbiology laboratory of the same hospital and analyzed within $24 \mathrm{~h}$. The stool samples were tested for $C$. difficile glutamate dehydrogenase (GDH) antigen and toxin using the C. DIFF QUIK CHEK COMPLETE® rapid test EIA kits (Techlab, Blacksburg, Virginia, USA) [2].

Stools were also sent in Cary Blair transport medium at ambient temperature to the Microbiology Department of PathWest Laboratory Medicine in Perth, Australia for further tests as described in Putsathit et al. [22]. The gold standard was toxigenic culture, and this was performed by direct plating the stool samples onto ChromID C. difficile agar (bioMérieux, Marcy l'Etoile, France). Selective enrichment was also undertaken in Robertson's cooked meat broth supplemented with gentamicin, cefoxitin and cycloserine and with addition of taurocholic acid as a germinant. All agar plates were incubated at $35^{\circ} \mathrm{C}$ in an A35 anaerobe chamber (Don Whitley Scientific, Otley, West Yorkshire) for up to $48 \mathrm{~h}$. Putative colonies of $C$. difficile were sub-cultured onto pre-reduced blood agar plates and identified by their morphology, horsedung odor and chartreuse (yellow-green) fluorescence under $360 \mathrm{~nm}$ UV light. Ambiguous colonies were further tested with Gram staining and the L-proline amino peptidase test.

All isolates were screened for $t c d A$ and $t c d B$ genes, binary toxin ( $c d t A$ and $c d t B$ ) genes and for changes in repeating region of $t c d A$ using PCR [23-25]. Products were run on the QIAxcel capillary electrophoresis platform and visualized on the QIAxcel ScreenGel software (Qiagen, Germany). Ribotyping was performed by PCR [26]. BioNumerics Software package v.6.5 (Applied Maths, Saint-Martens-Latem, Belgium) was used for dendrogram and cluster analysis of PCR ribotyping band patterns using Dice coefficient. PCR ribotyping banding patterns were identified by comparison of patterns with a reference library consisting of a collection of 30 reference strains from the European Centre for Disease Prevention and Control (ECDC), a collection of the most prevalent PCR RTs currently circulating in Australia (many of which are published), and a selection of binary toxin-positive strains which included RTs 027 and 078. Isolates that could not be assigned a RT were designated with internal nomenclature (prefix QX). Once all the tests were completed, stool samples were disposed in accordance to standard operating procedure of the laboratory.

\section{Significance of test results}

C. difficile was considered present if the GDH antigen and/or direct culture were positive. If the GDH antigen was negative but a toxigenic strain of $C$. difficile was isolated, the GDH result was deemed a false negative. A positive EIA result for toxin or isolation of toxigenic $C$. difficile was considered to indicate the presence of CDI.

\section{Ethical approval}

These studies were approved by the Universiti Sains Malaysia (USM) human research and ethics committee (Ref. no: USM/JEPeM/14080295). Study consent from the relevant authorities (i.e. the directors from the hospital and the aged residential care facility, respectively) were also obtained for the studies prior to commencement.

\section{Statistical analysis}

For the hospital study, participants who had C. difficile were categorized either to non-toxigenic $C$. difficile colonization and CDI based on our laboratory analysis. Analysis was performed using IBM Statistical Package for Social Sciences (SPSS) version 20.0 (IBM Corp., New York, USA). Numerical variables were expressed as mean \pm standard deviation (s.D.) and the values of categorical variables were summarized by frequency and percentage. In the univariate analysis, $\chi^{2}$ was used to assess for independent variables associated with non-toxigenic $C$. difficile 
colonization and CDI. A $P$-value $<0.05$ was used to indicate good fitting of the models.

\section{RESULTS}

\section{Hospital study}

In total, 76 participants (mean age $45 \pm 19$ years, 50\% females) were recruited and $29 \%$ of them $(n=22)$ were elderly (i.e. $\geqslant 60$ years). By EIA, $26 \%$ of the 76 stool samples $(n=20)$ were positive for GDH, and of the 20 positive samples, $9 \%(n=7)$ were positive for toxin. By culture, 19 (16 positive by direct culture, additional three positive by enrichment only) out of 76 stool samples (25\%) were positive for $C$. difficile, seven of which were toxigenic $(7 \%)$. Overall, the prevalence of CDI and non-toxigenic $C$. difficile colonization was $13 \%$ $(n=10)$ and $16 \%(n=12)$, respectively, and among the elderly, 4\% $(n=3)$ and $7 \%(n=5)$, respectively. There was no significant difference between nontoxigenic $C$. difficile colonization and CDI in prevalence for the overall group $(P=0 \cdot 67)$ and the elderly $(P=0 \cdot 48)$.

When compared to toxigenic culture (i.e. culture followed by $t c d B$ PCR) as the reference method, the sensitivity, specificity and negative predictive value of the EIA for GDH were 0.84 (95\% confidence interval (CI) 0.59-0.96), 0.93 (0.82-0.97), 0.95 (0.84-0.99), respectively. When compared to toxigenic culture, the sensitivity, specificity and negative predictive value of toxin detection were $0 \cdot 32$ (CI $0 \cdot 14-0 \cdot 57$ ), 0.98 (CI 0.89-0.99), 0.81 (CI 0.69-0.89), respectively.

Of the 19 positive cultures, two distinct isolates were identified in three specimens giving a total of 22 strains. Of these 22 strains, RT $043(14 \%)$ and RT 017 (14\%) were the most common (Table 1).

History of prior use of antibiotics was significantly associated with non-toxigenic $C$. difficile colonization $(P<0 \cdot 001)$ and CDI $(P<0 \cdot 001)$. The most commonly used antibiotics were penicillin $(37 \%)$, followed by cephalosporin $(29 \%)$, carbapenem $(15 \%)$, macrolide $(5 \%)$, metronidazole $(4 \%)$, fluoroquinolones $(4 \%)$, aminoglycoside $(1 \%)$ and vancomycin $(1 \%)$. Of these antibiotics, non-toxigenic $C$. difficile colonization was associated with penicillin and carbapenem (both $P<0.05)$ and CDI with penicillin and cephalosporin (both $P<0.05$ ). A proton pump inhibitor (PPI) was prescribed for $40 \%$ of participants and a history of prior use of a PPI was significantly associated with non-toxigenic $C$. difficile colonization $(P=0.02)$ and CDI $(P=0 \cdot 02)$.
Table 1. Summary of Clostridium difficile PCR ribotypes and toxin profiles in hospitalized participants

\begin{tabular}{lllll}
\hline \hline & \multicolumn{3}{l}{ Toxin profile } & \\
\cline { 2 - 4 } PCR ribotype & $t c d A$ & $t c d B$ & $c d t A / B$ & $n(\%)$ \\
\hline 043 & + & + & - & $3(14)$ \\
017 & - & + & - & $3(14)$ \\
QX002 & - & - & - & $2(9)$ \\
QX083 & - & - & - & $2(9)$ \\
014 group & - & - & - & $1(5)$ \\
QX567 & - & - & - & $1(5)$ \\
QX563 & - & - & - & $1(5)$ \\
QX117 & - & - & - & $1(5)$ \\
QX464 & - & - & - & $1(5)$ \\
QX463 & - & - & - & $1(5)$ \\
QX474 & + & + & - & $1(5)$ \\
QX107 & - & - & - & $1(5)$ \\
QX542 & - & - & - & $1(5)$ \\
QX564 & - & - & - & $1(5)$ \\
QX566 & - & - & - & $1(5)$ \\
Unknown & - & - & - & $1(5)$ \\
& & & Total & 22 \\
\hline \hline
\end{tabular}

Table 2 shows risk factors and clinical presentations associated with the prevalence of $C$. difficile. Duration of hospitalization, presence of underlying medical illnesses and increasing Charlson Comorbidity Index were significantly associated with non-toxigenic C. difficile colonization (all $P<0 \cdot 05$ ). On the other hand, duration of hospitalization and use of nasogastric tube were significantly associated with CDI (all $P<0 \cdot 05$ ). Fever (temperature $>38^{\circ} \mathrm{C}$ ) was the only symptom that was significantly associated with CDI $(P=0 \cdot 015)$. Of the clinical parameters, lower mean of albumin and higher CRP levels were significantly associated with both non-toxigenic $C$. difficile colonization (all $P<0 \cdot 05$ ) and CDI (all $P<0 \cdot 05$ ).

\section{Community study}

With a high prevalence of $C$. difficile found among elderly in the hospital study, we continued with the community study to observe if there was a similar trend. There were 138 elderly subjects who consented to the study and $78 \%(n=108)$ were of ethnic Malays. There was an equal distribution of gender between participants (females 51\% $(n=71)$ and males $49 \%$ $(n=67))$. The mean age was $72 \pm 8$ years. Only $6 \%$ of participants $(n=8)$ used a PPI and $3 \%$ of participants $(n=4)$ had a recent hospital admission within 12 weeks. Of $4 \%$ of participants $(n=5)$ who reported the use of antibiotics, most $(80 \%)$ took penicillin followed 
Table 2. Comparison between prevalence of Clostridium difficile with risk factors and clinical presentations in hospitalized participants

\begin{tabular}{|c|c|c|c|c|c|c|}
\hline \multirow[b]{2}{*}{ Variables } & \multicolumn{2}{|c|}{$\begin{array}{l}\text { Non-toxigenic } C \text {. difficile } \\
\text { colonization }(n=66)^{\mathrm{a}}\end{array}$} & \multirow[b]{2}{*}{$P$} & \multicolumn{2}{|l|}{$\mathrm{CDI}(n=64)^{\mathrm{b}}$} & \multirow[b]{2}{*}{$P$} \\
\hline & $\begin{array}{l}\text { Positive } \\
(n=12)\end{array}$ & $\begin{array}{l}\text { Negative } \\
(n=54)\end{array}$ & & $\begin{array}{l}\text { Positive } \\
(n=10)\end{array}$ & $\begin{array}{l}\text { Negative } \\
(n=54)\end{array}$ & \\
\hline \multicolumn{7}{|l|}{ Risk factors } \\
\hline Age (yrs) & $52 \cdot 0 \pm 18 \cdot 8^{\mathrm{c}}$ & $42 \cdot 7 \pm 18 \cdot 6$ & $0 \cdot 124$ & $49 \cdot 6 \pm 18 \cdot 2$ & $42 \cdot 7 \pm 18 \cdot 6$ & $0 \cdot 286$ \\
\hline \multicolumn{7}{|c|}{ Length of hospitalization (wk), $n(\%)^{\mathrm{d}}$} \\
\hline$<1$ & $1(8)$ & $44(82)$ & \multirow[t]{2}{*}{$<0.001$} & $0(0)$ & $44(82)$ & \multirow[t]{2}{*}{$<0.001$} \\
\hline$\geqslant 1$ & $11(92)$ & $10(19)$ & & $10(100)$ & $10(19)$ & \\
\hline \multicolumn{7}{|l|}{ Nasogastric tube, $n(\%)^{\mathrm{a}}$} \\
\hline Yes & $2(17)$ & $2(4)$ & \multirow[t]{2}{*}{$0 \cdot 148$} & $4(40)$ & $2(4)$ & \multirow[t]{2}{*}{$0 \cdot 004$} \\
\hline No & $10(83)$ & $52(96)$ & & $6(60)$ & $52(96)$ & \\
\hline \multicolumn{7}{|l|}{ Medical illness } \\
\hline Yes & $11(92)$ & $27(50)$ & \multirow[t]{2}{*}{$0 \cdot 009$} & $7(70)$ & $27(50)$ & \multirow[t]{2}{*}{$0 \cdot 313$} \\
\hline No & $1(8)$ & $27(50)$ & & $3(30)$ & $27(50)$ & \\
\hline Charlson Comorbidity Index & $2 \cdot 50 \pm 1 \cdot 51$ & $0 \cdot 96 \pm 1 \cdot 12$ & $<0.001$ & $2 \cdot 20 \pm 1 \cdot 93$ & $0 \cdot 96 \pm 1 \cdot 12$ & $0 \cdot 077$ \\
\hline \multicolumn{7}{|l|}{ Symptoms } \\
\hline \multicolumn{7}{|l|}{ Abdominal pain, $n(\%)$} \\
\hline Yes & $4(33)$ & $8(15)$ & \multirow[t]{2}{*}{$0 \cdot 208$} & $4(40)$ & $8(15)$ & \multirow[t]{2}{*}{$0 \cdot 082$} \\
\hline No & $8(67)$ & $46(85)$ & & $6(60)$ & $46(85)$ & \\
\hline \multicolumn{7}{|l|}{ Fever, $n(\%)$} \\
\hline Yes & $8(68)$ & $25(46)$ & \multirow[t]{2}{*}{$0 \cdot 339$} & $9(90)$ & $25(46)$ & \multirow[t]{2}{*}{$0 \cdot 015$} \\
\hline No & $4(32)$ & $29(54)$ & & $1(10)$ & $29(54)$ & \\
\hline \multicolumn{7}{|l|}{ Clinical parameters } \\
\hline $\mathrm{WBC}, \times 10^{9} / 1$ & $8 \cdot 51 \pm 7 \cdot 06$ & $7 \cdot 15 \pm 4 \cdot 17$ & 0.530 & $9 \cdot 73 \pm 5 \cdot 67$ & $7 \cdot 15 \pm 4 \cdot 17$ & 0.095 \\
\hline Urea, mmol/l & $10 \cdot 19 \pm 8 \cdot 77$ & $4 \cdot 97 \pm 3.05$ & $0 \cdot 065$ & $8 \cdot 08 \pm 10 \cdot 06$ & $4 \cdot 97 \pm 3.05$ & $0 \cdot 262$ \\
\hline Creatinine, $\mu \mathrm{mol} / \mathrm{l}$ & $240 \cdot 83 \pm 233 \cdot 68$ & $96 \cdot 15 \pm 46 \cdot 07$ & $0 \cdot 056$ & $251 \cdot 30 \pm 530 \cdot 45$ & $96 \cdot 15 \pm 46 \cdot 07$ & 0.379 \\
\hline Albumin, $\mathrm{g} / 1$ & $27 \cdot 58 \pm 8 \cdot 31$ & $34 \cdot 39 \pm 5 \cdot 07$ & 0.017 & $30 \cdot 00 \pm 6 \cdot 68$ & $34 \cdot 39 \pm 5 \cdot 07$ & 0.020 \\
\hline $\mathrm{CRP}, \mathrm{mg} / \mathrm{l}$ & $111 \cdot 08 \pm 59 \cdot 04$ & $63 \cdot 44 \pm 62 \cdot 90$ & 0.019 & $112 \cdot 40 \pm 54 \cdot 90$ & $63 \cdot 44 \pm 62 \cdot 90$ & 0.025 \\
\hline
\end{tabular}

Figures with significant $\mathrm{p}$ values are indicated in bold. CRP, C-reactive protein; WBC, white blood cells; wk, weeks; yrs, years. ${ }^{a}$ CDI cases were excluded from the non-toxigenic $C$. difficile colonization total.

${ }^{\mathrm{b}}$ Non-toxigenic $C$. difficile colonization cases were excluded from the CDI total.

${ }^{\mathrm{c}}$ Mean \pm S.D. (all such values).

${ }^{\mathrm{d}}$ Due to rounding, the total may not add to $100 \%$ exactly.

by macrolide (20\%). Most participants (94\%) did not have symptoms. Only 1\% $(n=2), 2 \%(n=3)$ and $3 \%$ $(n=4)$ of participants reported that they had experience of abdominal pain, diarrhea and fever, respectively.

Of the 138 stool samples, nine were discarded because they were inadequate, leaking or overflowing. The remaining 129 samples were analyzed. The prevalence of GDH, toxin by EIA and $C$. difficile by direct culture was $2 \%(n=2), 0 \%(n=0)$ and $2 \%(n=2)$, respectively. The two non-toxigenic $C$. difficile isolates were typed as QX 083 and QX 565.

\section{Awareness survey}

There were 154 participants who were surveyed. The mean age of participants was $35 \pm 7$ years and almost two-thirds were females $(66 \% ; n=102)$. Sixty percent of participants $(n=92)$ were clinicians. Of 154 participants, $33 \%(n=51), 14 \%(n=22), 4 \%(n=6)$ and $3 \%$ $(n=4)$ of respondents answered one, two, three or four awareness questions correctly, respectively. The remaining $46 \%$ of participants $(n=71)$ provided wrong answers for all the four awareness questions. For the fifth question, there were $20 \%$ participants $(n=31)$ reported $C$. difficile as underestimated in their practice and $31 \%(n=47)$ of participants reported not sure.

\section{DISCUSSION}

Our hospital prevalence of CDI was similar to previous reported studies from South East Asia [19, 27, 28], but 
we also found a significant rate of non-toxigenic C. difficile colonization not commonly reported in other Asian populations [29]. A recent study from Thailand [29] reported a similar prevalence of nontoxigenic $C$. difficile colonization $(16 \%)$ as in our study. The authors suggested that the high prevalence of non-toxigenic $C$. difficile colonization may play an important role in reducing the risk of developing CDI [29]. This is because patients with non-toxigenic C. difficile colonization may mount a sufficient immune response to $C$. difficile toxins [8]. There are limited data on non-toxigenic $C$. difficile colonization in Asia countries because most molecular epidemiological studies on the prevalence of $C$. difficile only focused on the characterization and isolation of the toxigenic $C$. difficile strains associated with CDI [14]. Therefore, the prevalence and molecular epidemiology of non-toxigenic strains may not have been properly investigated and documented [29].

In our study, 22 different strains were identified with RTs 043 and 017 being the most common. Prior to our study, there are no published data on C. difficile ribotyping in Malaysia. According to available data, the most prevalent RTs in Asia include 001, 002, 014, 017 and 018 [14], but our study also reported other new and unknown RTs. Although RT 014/020 is usually toxigenic, we found some unusual isolates of RT 014/020 that are non-toxigenic, which has been reported from Thailand previously [29]. Furthermore, the epidemic RTs 027 and 078 were not identified in our study. These findings suggest that there is an undefined and widely distributed reservoir of $C$. difficile strains in Malaysia. It is likely also that the $C$. difficile strains circulating in Malaysia are different from that in North America and Europe. The above suggests that CDI is as much a problem in South East Asia as elsewhere in the world; however, the molecular epidemiology may be specific for the country and the region.

In our hospital study, non-toxigenic $C$. difficile colonization and CDI shared some risk factors, including history of prior use of antibiotics, history of prior use of PPI, length of hospitalization, albumin and C-reactive protein. All these risk factors were known risk factors for $C$. difficile, which are consistent with previous studies [30-34]. The prescription of antibiotic in our setting is mainly based on the Malaysian National Antibiotic Guidelines 2014, which is not much different from other guidelines from the Western countries. The common prescription of high doses of broad-spectrum antibiotics and possible resistance to metronidazole in our hospital may explain the high prevalence of CDI observed in our study [28]. Broad-spectrum antibiotic causes significant disruption of the gut microbiome and alter the gut ecology [35]. A disturbed gut ecology allows C. difficile to proliferate and causes infection in a susceptible human host [36].

Since high prevalence of CDI in elderly in the hospital is a relatively common finding, we conducted the community study in an elderly population to determine if there would be a similar trend of $C$. difficile in the community as in the hospital. However, our study reported a low prevalence of $C$. difficile carriage $(1 \cdot 6 \%)$ in the elderly community and none of the elderly had CDI. The low prevalence in our elderly community may be due to several reasons, especially the little or no contacts with the hospital environment and the absence of other traditional risk factors of CDI, in particular the low usage of antibiotics.

In addition, little or no exposure of PPI in our elderly community may explain the low prevalence of C. difficile observed in this population. The prevalence in our community study was also low when compared with other studies that reported asymptomatic colonization between $6 \%$ and $50 \%$ in long-term care facility residents [5]. A recent meta-analysis of nine studies reported that $15 \%$ of long-term care facility residents were asymptomatic carriers of toxigenic $C$. difficile [37]. It is possible that cleaning and disinfection, or appropriate hand hygiene in the hospital might not be adequate, allowing spores to be transmitted frequently in the hospital.

Our awareness study reported a low level of awareness with regards to CDI among hospital staff and students. The low awareness means a low CDI detection rate in our hospital and subsequently causes delays in the provision of antibiotics. Our findings were similar with an international survey that reported only $2 \%$ of respondents answered all questions correctly and $44 \%$ reported $C$. difficile to be underestimated [15]. The international survey was conducted among clinicians from North America (55\%), Europe (32\%), Asia/ Pacific (12\%), Central and South America (1\%), and Africa (1\%) [15]. Mavros et al. [15] reported that clinicians from Asia had a significantly lower score than those from North America, suggesting that there was an inadequate awareness level of CDI among clinicians, particularly in Asia.

Our study had strengths but also limitations. Our study generated novel local data regarding the prevalence of CDI in hospitalized participants and the elderly in the community. In addition, we also 
investigated awareness of CDI among healthcare personnel and the low awareness shown in our study may mean that the hospital is missing many CDI cases and therefore new policy measures are urgently needed. There was a high carriage rate of non-toxigenic strains among hospitalized participants, and it is possible that some cases where toxigenic $C$. difficile was isolated may have been colonized rather than infected. RTs 017 and 043 were the most commonly found RTs in our hospital; however, there was also a significant number of new and unknown RTs, which may be unique to the population in Asia. Further investigation is needed to determine the significance of RT diversity with high rates of non-toxigenic strains. However, currently only a limited number of centers around the world, mainly in Europe, carry out ribotyping routinely so the assigning of new RTs is a slow process. Unfortunately, there are no recognized reference centers in Asia, and this will be required before the development of a useable Asian database.

In conclusion, our study demonstrates that the high prevalence of CDI in a Malaysian hospital is similarly reported by other Asian countries, despite a high prevalence of non-toxigenic $C$. difficile colonization. Important risk factors for CDI include duration of hospitalization and use of antibiotics. Although $C$. difficile colonization was highly prevalent in the hospital setting, $C$. difficile colonization was rare among the elderly in the community largely due to the absence of hospital exposure and absence of other traditional risk factors including antibiotics and PPI. The alarmingly low awareness of CDI among Malaysia hospital staffs needs to be addressed.

\section{ACKNOWLEDGEMENTS}

This study was partly funded by an Exploratory Research Grant Scheme (ERGS) from the Ministry of Higher Education of Malaysia (USM/203/PPSP/ 6730022). This work was also supported by a grant from the University of Western Australia and Alere.

The authors are extremely grateful to all the subjects who took part in the study.

\section{AUTHOR CONTRIBUTIONS}

N. H. Zainul, A. Besari, S. A. Hassan, D. A. Collins, T. V. Riley and Y. Y. Lee participated in study recruitment and study design. R. A. Rahman, D. A. Collins and T. V. Riley were involved in the preparation and analysis of the biological samples. N. H. Zainul,
Z. F. Ma, N. Hamid and Y. Y. Lee conducted statistical analysis and interpreted the data. N. H. Zainul and Z. F. Ma wrote the first draft of the manuscript. Z. F. Ma and Y. Y. Lee revised the manuscript. All authors read and approved the final version of the manuscript.

\section{DECLARATION OF INTEREST}

None.

\section{REFERENCES}

1. Carter GP, Rood JI, Lyras D. The role of toxin A and toxin $\mathrm{B}$ in the virulence of Clostridium difficile. Trends in Microbiology 2012; 20(1): 21-29.

2. Eckert $\mathbf{C}$, et al. Prevalence and pathogenicity of binary toxin-positive Clostridium difficile strains that do not produce toxins A and B. New Microbes and New Infections 2015; 3: 12-17.

3. Martin JSH, Monaghan TM, Wilcox MH. Clostridium difficile infection: epidemiology, diagnosis and understanding transmission. Nature Reviews Gastroenterology \& Hepatology 2016; 13(4): 206-216.

4. Ghose C. Clostridium difficile infection in the twenty-first century. Emerging Microbes \& Infections 2013; 2: e62.

5. Sartelli M, et al. WSES guidelines for management of Clostridium difficile infection in surgical patients. World Journal of Emergency Surgery 2015; 10: 38.

6. Heinlen L, Ballard JD. Clostridium difficile infection. The American Journal of the Medical Sciences 2010; 340(3): 247-252.

7. Barbut F, Jones G, Eckert C. Epidemiology and control of Clostridium difficile infections in healthcare settings: an update. Current Opinion in Infectious Diseases 2011; 24(4): 370-376.

8. Furuya-Kanamori L, et al. Asymptomatic Clostridium difficile colonization: epidemiology and clinical implications. BMC Infectious Diseases 2015; 15: 516.

9. Lo Vecchio A, Zacur GM. Clostridium difficile infection: an update on epidemiology, risk factors, and therapeutic options. Current Opinion in Gastroenterology 2012; 28 (1): 1-9.

10. Gilca R, et al. Epidemiological patterns and hospital characteristics associated with increased incidence of Clostridium difficile infection in Quebec, Canada, 1998-2006. Infection Control \& Hospital Epidemiology 2010; 31(9): 939-947.

11. Vedantam G, et al. Clostridium difficile infection: toxins and non-toxin virulence factors, and their contributions to disease establishment and host response. Gut Microbes 2012; 3(2): 121-134.

12. Carter GP, et al. The anti-sigma factor TcdC modulates hypervirulence in an epidemic BI/NAP1/027 clinical isolate of Clostridium difficile. PLoS Pathogens 2011; 7(10): e1002317.

13. Crobach MJT, et al. European society of clinical microbiology and infectious diseases: update of the diagnostic 
guidance document for Clostridium difficile infection. Clinical Microbiology \& Infection 2016; 22: S63-S81.

14. Collins DA, Hawkey PM, Riley TV. Epidemiology of Clostridium difficile infection in Asia. Antimicrobial Resistance \& Infection Control 2013; 2(1): 21.

15. Mavros MN, et al. Underestimation of Clostridium difficile infection among clinicians: an international survey. European Journal of Clinical Microbiology \& Infectious Diseases 2012; 31(9): 2439-2444.

16. Boey CC, Ramanujam TM, Looi LM. Clostridium difficile-related necrotizing pseudomembranous enteritis in association with Henoch-Schonlein purpura. Journal of Pediatric Gastroenterology \& Nutrition 1997; 24(4): 426-429.

17. Hassan H, Cheng SC. Detection of Clostridium difficile from an enhancement broth by gas-liquid chromatography. The Malaysian Journal of Pathology 1991; 13(1): 29-31.

18. Parasakthi N, et al. Clostridium difficile associated diarrhoea: a report of seven cases. Singapore Medical Journal 1988; 29(5): 504-507.

19. Hassan SA, et al. Prevalence of Clostridium difficile toxin in diarhoeal stool samples of patients from a tertiary hospital in North Eastern Peninsular Malaysia. The Medical Journal of Malaysia 2012; 67(4): 402-405.

20. Syuhada N, et al. Clostridium difficile infection: clinicoepidemiological perspective. Medicine \& Health 2013; 8(2): 64-72.

21. McDonald LC, et al. Recommendations for surveillance of Clostridium difficile-associated disease. Infection Control and Hospital Epidemiology 2007; 28(2): 140-145.

22. Putsathit $\mathbf{P}$, et al. Evaluation of the BD Max Cdiff assay for the detection of toxigenic Clostridium difficile in human stool specimens. Pathology 2015; 47(2): 165-168.

23. Kato $\mathbf{N}$, et al. Identification of toxigenic Clostridium difficile by the polymerase chain reaction. Journal of Clinical Microbiology 1991; 29(1): 33-37.

24. Kato H, et al. Identification of toxin A-negative, toxin B-positive Clostridium difficile by PCR. Journal of Clinical Microbiology 1998; 36(8): 2178-2182.

25. Stubbs S, et al. Production of actin-specific ADPribosyltransferase (binary toxin) by strains of Clostridium difficile. FEMS Microbiology Letters 2000; 186(2): 307-312.

26. Stubbs SL, et al. PCR targeted to the 16S-23S rRNA gene intergenic spacer region of Clostridium difficile and construction of a library consisting of 116 different PCR ribotypes. Journal of Clinical Microbiology 1999; 37(2): 461-463.

27. Putsathit P, et al. Clostridium difficile infection in Thailand. International Journal of Antimicrobial Agents 2015; 45(1): 1-7.

28. Hassan SA, et al. Hospital-acquired Clostridium difficile infection among patients with type 2 diabetes mellitus in acute medical wards. The Journal of the Royal College of Physicians of Edinburgh 2013; 43(2): 103-107.

29. Putsathit P, et al. Prevalence and molecular epidemiology of Clostridium difficile infection in Thailand. New Microbes and New Infections 2017; 15: 27-32.

30. Al-Tureihi FIJ, et al. Albumin, length of stay, and proton pump inhibitors: key factors in Clostridium difficileassociated disease in nursing home patients. Journal of the American Medical Directors Association 2005; 6(2): 105-108.

31. Dubberke ER, et al. Risk factors for acquisition and loss of Clostridium difficile colonization in hospitalized patients. Antimicrobial Agents \& Chemotherapy 2015; 59(8): 4533-4543.

32. Alasmari F, et al. Prevalence and risk factors for asymptomatic Clostridium difficile carriage. Clinical Infectious Diseases 2014; 59(2): 216-222.

33. McPherson S, et al. Intravenous immunoglobulin for the treatment of severe, refractory, and recurrent Clostridium difficile diarrhea. Diseases of the Colon \& Rectum 2006; 49(5): 640-645.

34. Hardt $\mathbf{C}$, et al. Univariate and multivariate analysis of risk factors for severe Clostridium difficile-associated diarrhoea: importance of co-morbidity and serum C-reactive protein. World Journal of Gastroenterology 2008; 14(27): 4338-4341.

35. Francino MP. Antibiotics and the human gut microbiome: dysbioses and accumulation of resistances. Frontiers in Microbiology 2015; 6: 1543.

36. Langdon A, Crook N, Dantas G. The effects of antibiotics on the microbiome throughout development and alternative approaches for therapeutic modulation. Genome Medicine 2016; 8: 39.

37. Ziakas PD, et al. Asymptomatic carriers of toxigenic C. difficile in long-term care facilities: a meta-analysis of prevalence and risk factors. PLOS ONE 2015; 10(2): $\mathrm{e} 0117195$. 